PROCEEDINGS OF THE

AMERICAN MATHEMATICAL SOCIETY

Volume 139, Number 1, January 2011, Pages 293-303

S 0002-9939(2010)10507-5

Article electronically published on July 22, 2010

\title{
A RIEMANNIAN FOUR VERTEX THEOREM FOR SURFACES WITH BOUNDARY
}

\author{
MOHAMMAD GHOMI
}

(Communicated by Jon G. Wolfson)

\begin{abstract}
We prove that every metric of constant curvature on a compact surface $M$ with boundary $\partial M$ induces at least four vertices, i.e., local extrema of geodesic curvature on a connected component of $\partial M$ if, and only if, $M$ is simply connected. Indeed, when $M$ is not simply connected, we construct hyperbolic, parabolic, and elliptic metrics of constant curvature on $M$ with only two critical points of geodesic curvature on each component of $\partial M$. With few exceptions, these metrics are obtained by removing the singularities and a perturbation of flat structures on closed surfaces.
\end{abstract}

\section{INTRODUCTION}

This work is concerned with the relation between the topology of a compact Riemannian surface and the number of the critical points of geodesic curvature of its boundary. The motivation for this problem may be traced back to the classical theorem of Kneser [13, 11, published in 1912, which states that any simple closed curve in the Euclidean plane must have at least four vertices, i.e., local extrema of geodesic curvature. In 1987 Pinkall [19] extended Kneser's theorem to closed curves which bound compact immersed surfaces, and it has been shown that this result holds in any simply connected 2-dimensional space form [4, 14. One might then wonder if these observations indicate a deeper phenomenon, i.e., whether the boundary of any compact surface of constant curvature must have four vertices. Here we show that the answer depends on the underlying topology:

Theorem 1.1. Let $M$ be a compact surface with boundary $\partial M$. Then every metric of constant curvature on $M$ induces at least four vertices on a connected component of $\partial M$, if, and only if, $M$ is simply connected. Indeed, if $M$ is not simply connected, then for any constant $K$ there exists a metric of constant curvature $K$ on $M$ with only two critical points of geodesic curvature on each component of $\partial M$.

One direction of the proof of this result follows quickly from the fact that any disk of constant curvature may be developed in a simply connected space form (Section 2). The other direction, which is the main feature of this work, is proved by constructing flat surfaces of every nontrivial topological type with only two

Received by the editors September 30, 2009 and, in revised form, March 19, 2010.

2010 Mathematics Subject Classification. Primary 53C20, 53C22; Secondary 53A04, 53A05.

Key words and phrases. Four vertex theorems, flat structures with conical singularities, surfaces of constant curvature with boundary, space forms, geodesic vertices.

The author was supported by NSF Grant DMS-0336455, and CAREER award DMS-0332333.

(C)2010 American Mathematical Society Reverts to public domain 28 years from publication 
critical points of geodesic curvature, which are both nondegenerate, on each of their boundary components (Section 3). In most cases, these metrics are obtained by removing conical singularities of flat structures on closed surfaces, but some low genus cases need to be treated separately. Then we show that any compact flat surface with boundary may be perturbed, in the $\mathcal{C}^{\infty}$ sense, to have constant positive or negative curvature (Section 4), and thus we obtain elliptic and hyperbolic versions of our flat surfaces.

Since it makes no reference to an ambient space, Theorem 1.1 may be considered a Riemannian version of the classical four vertex theorem. One might also contrast this result with the uniformization theorem of Osgood, Phillips, and Sarnak [17, which yields metrics of constant curvature on compact surfaces with constant geodesic curvature on their boundaries (so every boundary point becomes a vertex). Other methods for producing constant curvature metrics on surfaces with boundary include curvature flow [2], the $h$-principle [7, Thm 1.2], and Monge-Ampère equations [9, 28], although none of these results are concerned with minimizing the number of vertices. Four vertex theorems have had an interesting history, dating back to the first version of the theorem proved by Mukhopadhyaya [16] for convex curves in 1905, which was later generalized to simple curves by Kneser [13. For surveys of results in this area and some recent developments and applications see [5, 18, 30, 24, 6, 22, Singular flat structures on surfaces have been extensively studied due to their connections with Teichmüller theory and other applications; see [26, 15, 25] for some overview and further references.

Note 1.2. It is easy to construct noncompact surfaces of constant curvature with only two vertices on their boundary. For instance consider the curve $\Gamma$ traced by $(\cos (t), \sin (t), \sin (t))$ on the cylinder $C:=\mathbf{S}^{1} \times \mathbf{R} \subset \mathbf{R}^{3}$, and let $M$ be the portion of $C$ above $\Gamma$. Then $M$ is a metrically complete surface of constant curvature with only two vertices on its boundary. Constructing compact examples, on the other hand, which is the main point of Theorem 1.1, will take more effort.

Note 1.3. Theorem 1.1 does not remain true if the curvature is not constant; i.e., there are metrics with nonconstant curvature on the disk which induce only two vertices on the boundary. This follows, for instance, from a theorem of Jackson 12, who showed that if $p$ is any nonstationary point of curvature in a Riemannian surface, then small metric circles centered at $p$ have only two vertices.

Note 1.4. There is no obvious analogue for Theorem 1.1 in higher dimensions. For instance, there are flat metrics on the 3-dimensional ball which induce only two vertices, i.e., local extrema of Gauss curvature, on the boundary of the ball. This follows from Gluck's generalization of Minkowski's theorem [8] which states that any smooth positive function on $\mathbf{S}^{n-1}$ (including those with only two critical points) may be realized as the Gauss-Kronecker curvature of a convex hypersurface in $\mathbf{R}^{n}$.

Similarly, one may construct closed surfaces embedded in Euclidean space with only two critical points of mean curvature. Indeed, sufficient conditions [20, p. 442] for the existence of such a surface in $\mathbf{R}^{3}$ with prescribed mean curvature $H$ as a function of outward normal $u \in \mathbf{S}^{2}$ are that (i) $H \geq 0$; (ii) $H \geq H_{s s}$, where $H_{s s}$ denotes the second derivative of $H$ with respect to arclength along great circles; and (iii) $\int_{\mathbf{S}^{2}} u H(u)=0$. It remains then to check that there exists a function $H: \mathbf{S}^{2} \rightarrow \mathbf{R}$ with only two critical points which satisfies these conditions. To this end, start with any positive function $f: \mathbf{S}^{2} \rightarrow \mathbf{R}$ with only two critical points. By 
a theorem of Gluck [8] there exists a diffeomorphism $\phi: \mathbf{S}^{2} \rightarrow \mathbf{S}^{2}$ such that $f \circ \phi$ meets condition (iii). Finally set $H:=f \circ \phi+C$ where $C$ is a sufficiently large constant. Then condition (ii) will also be satisfied.

\section{The Simply CONNECTED CASE}

Here we show that if the surface $M$ of Theorem 1.1] is simply connected (i.e., it is a disk), then every metric of constant curvature on $M$ induces at least four vertices on $\partial M$. We begin by recalling the generalized version of Pinkall's theorem. Unless stated otherwise, we assume that all curves and surfaces in this paper are smooth, i.e., $\mathcal{C}^{\infty}$.

Lemma 2.1 (19, 14, 4). Any closed curve which bounds a compact immersed surface in a simply connected 2-dimensional space form has at least four vertices.

Proof. After a rescaling, we may assume that the space form is the Euclidean plane $\mathbf{R}^{2}$, the sphere $\mathbf{S}^{2}$, or the hyperbolic plane $\mathbf{H}^{2}$. Once this result is proved for $\mathbf{R}^{2}$ (see [19, 29, 3 for different ways to do this), then the case of $\mathbf{S}^{2}$ follows quickly since stereographic projection preserves vertices, as was already known to Kneser 13]; see [19, 14. The hyperbolic case follows similarly after we identify $\mathbf{H}^{2}$ with the Poincaré disk $D \subset \mathbf{R}^{2}$ and observe that the inclusion map $D \hookrightarrow \mathbf{R}^{2}$ preserves vertices [14.

Now, to prove the "if" part of Theorem 1.1] it suffices to note the following basic fact, which is well known to those familiar with developing maps for $(X, G)$ manifolds [21, 1]. We include, however, a simple proof for completeness.

Lemma 2.2. Any disk of constant curvature may be isometrically immersed in a simply connected 2-dimensional space form.

Proof. We may cover the given disk, say $D$, by a sequence of open neighborhoods $U_{i}$, $i=1, \ldots, N$, of arbitrary small radius and with the property that $\left(\bigcup_{i=1}^{k} U_{i}\right) \cap U_{k+1}$ is connected and nonempty, for $1 \leq k \leq N-1$. This may be done as follows: identify $D$ with the square $S:=[0,1] \times[0,1]$ in $\mathbf{R}^{2}$; divide $S$ into the grid of subsquares $S_{i j}:=[(i-1) / n, i / n] \times[(j-1) / n, j / n], i, j=1, \ldots, n$; let $\widetilde{S}_{i j}$ be a slight enlargement of each $S_{i j}$ consisting of points in $D$ which are within a distance $\epsilon$ of $S_{i j}$ for some $0<\epsilon<1 / 2 n$; and, finally, define $U_{i}$ by a renumbering of $\widetilde{S}_{i j}$ according to the "snaking" sequence $\widetilde{S}_{11}, \ldots, \widetilde{S}_{1 n}, \widetilde{S}_{2 n}, \ldots \widetilde{S}_{21}, \widetilde{S}_{31}, \ldots, \widetilde{S}_{3 n}, \ldots$

If $X$ is a space form with the same curvature as $D$, then for each $U_{i}$ there exists an isometric embedding $f_{i}: U_{i} \rightarrow X$ (assuming that $U_{i}$ are sufficiently small or $n$ is large). We define $f: D \rightarrow X$ by an inductive gluing of $f_{i}$ as follows. Set $f:=f_{1}$ on $U_{1}$. Suppose $f$ is defined on $\bigcup_{i=1}^{k} U_{i}$. We claim that there exists an isometry $\rho: X \rightarrow X$ such that $\rho \circ f_{k+1}=f$ on $V:=\left(\bigcup_{i=1}^{k} U_{i}\right) \cap U_{k+1}$. Then we may set $f:=\rho \circ f_{k+1}$ on $U_{k+1}$, so $f$ will be well-defined on $\bigcup_{i=1}^{k+1} U_{i}$.

To find $\rho$, let $p \in V$, and let $e_{i}, i=1,2$, be an orthonormal basis for $T_{p} V$. Then $d f_{p}\left(e_{i}\right)$ and $\left(d f_{k+1}\right)_{p}\left(e_{i}\right)$ are orthonormal bases for $T_{f(p)} X$ and $T_{f_{k+1}(p)} X$ respectively. Since $X$ is homogeneous and isotropic, there exists an isometry $\rho: X \rightarrow X$ which maps $f_{k+1}(p)$ to $f(p)$ and $\left(d f_{k+1}\right)_{p}\left(e_{i}\right)$ to $d f_{p}\left(e_{i}\right)$. But $f \circ f_{k+1}^{-1}: f_{k+1}(V) \rightarrow$ $f(V)$ is also an isometry with these properties. Thus $\rho=f \circ f_{k+1}^{-1}$ on $f_{k+1}(V)$, 
since, due to uniqueness of geodesics, isometric embeddings from a connected manifold into another of the same dimension are determined by their differential at one point.

\section{Flat metrics With FeWESt Vertices}

Here we show that if $M$ in Theorem 1.1 is not simply connected, then it admits a flat metric, i.e., one with constant zero curvature, which induces on each connected component of $\partial M$ only two critical points of geodesic curvature. Furthermore, both critical points will be nondegenerate; i.e., the second derivative of the curvature will not vanish at these points. Suppose that $M$ has $k$ boundary components. Then $M$ is homeomorphic to a closed surface $\bar{M}$ minus $k$ open disks with disjoint closures, and $\chi(M)=\chi(\bar{M})-k$, where $\chi$ denotes the Euler characteristic. Also recall that if $\bar{M}$ is orientable, then it is homeomorphic to the connected sum of $g$ tori with the sphere $\mathbf{S}^{2}$, which yields that $\chi(\bar{M})=2-2 g$. On the other hand, when $\bar{M}$ is not orientable, then it is the connected sum of $g$ projective planes $\mathbf{R P}^{2}$, which yields that $\chi(\bar{M})=2-g$.

3.1. There are three special cases which we need to consider, before describing a general procedure for constructing our desired flat metrics in all other cases.

3.1.1. $(\chi(\bar{M})=2 \& k=2)$ If $M$ is an annulus, then the desired metric is obtained by gluing the vertical sides of the region in Figure 1. This region is bounded by

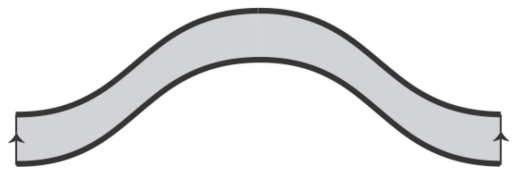

FIGURE 1

the curves $y=\cos (x) \pm c$ and $x= \pm \pi$. Note that the top and bottom curves each have three critical points of curvature, which occur at $x=0$ and $x= \pm \pi$; however, the latter points get identified after the gluing, and thus we end up with only two critical points on each boundary component.

3.1.2. $(\chi(\bar{M})=1 \& k=1)$ If $M$ is a Möbius strip, then we may take half of the region depicted in Figure 1, e.g., the portion between $x=0$ and $x=\pi$, and glue its right and left hand sides with a twist.

3.1.3. $(\chi(\bar{M})=0 \& k=1)$ If $M$ is a torus minus a disk, then the surface we seek may be constructed by gluing the sides of the region in Figure 2 and if $M$ is a Klein bottle minus a disk, then we just need to switch the direction of the arrows and the labels on the bottom side of this region. Note that the sum of the angles at all interior vertices is $2 \pi$. So the gluing yields a smooth flat surface with one boundary component, which is the dark curve in Figure 2 say $\gamma$, with its end points identified. The curvature of $\gamma$, as a function of arclength, is prescribed by

$$
\kappa(t)=1-\frac{3}{4} \cos (t)
$$

where $-\pi \leq t \leq \pi$. More explicitly, $\gamma(t):=\int_{0}^{t} e^{i \theta(s)} d s$, where $e^{i \theta}:=(\cos (\theta), \sin (\theta))$ and $\theta(t):=\int_{0}^{t} \kappa(s) d s$. Since the end points of $\gamma$ are orthogonal to side 3 of the 


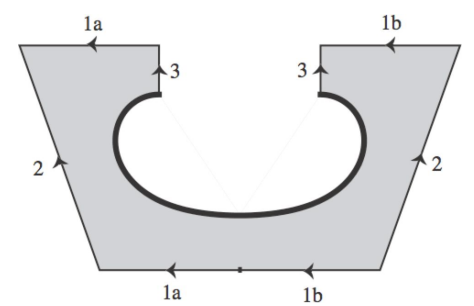

FiguRE 2

region in Figure 2. $\partial M$ will be $\mathcal{C}^{1}$. Further, since the end points of $\gamma$ have equal curvatures, it follows, by Lemma 3.1 below, that $\partial M$ is $\mathcal{C}^{2}$. So the curvature of $\partial M$ is given by $\kappa$. But the period of $\kappa$ coincides with the length of $\partial M$. Thus the curvature of $\partial M$ is $\mathcal{C}^{\infty}$, which implies that $\partial M$ is $\mathcal{C}^{\infty}$, again by Lemma 3.1 .

Lemma 3.1. Let $M$ be a Riemannian surface, $I=(-\epsilon, \epsilon)$ and $\gamma: I \rightarrow M$ be a $\mathcal{C}^{1}$-immersed curve which is $\mathcal{C}^{2}$ on $I-\{0\}$. Let $\kappa$ be the geodesic curvature of $\gamma$ on $I-\{0\}$, and suppose that $\lim _{t \rightarrow 0^{+}} \kappa(t)=\lim _{t \rightarrow 0^{-}} \kappa(t)$. Then $\gamma$ is $\mathcal{C}^{2}$. Furthermore, if $\kappa$ is $\mathcal{C}^{k}$ on $I$, then $\gamma$ is $\mathcal{C}^{k+2}$.

Proof. Since the problem is local, we may assume that $M$ is $\mathbf{R}^{2}$. Also we may assume that $\gamma$ has unit speed. Let $T:=\gamma^{\prime}$ and $N$ be a unit normal vector field along $\gamma$. Then, on $I-\{0\}$, we have $\kappa=\left\langle\gamma^{\prime \prime}, N\right\rangle$, and thus $\gamma^{\prime \prime}=\kappa N$. Since $T$ is continuous on $I$, so is $N$; therefore, $\lim _{t \rightarrow 0^{+}} \gamma^{\prime \prime}(t)=\lim _{t \rightarrow 0^{-}} \gamma^{\prime \prime}(t)$. So $\gamma^{\prime \prime}$ is continuous on $I$. Finally recall that, up to a rigid motion, $\gamma=\int e^{i \theta}$, where $\theta=\int \kappa$. Thus if $\kappa$ is $\mathcal{C}^{k}$, then $\gamma$ is $\mathcal{C}^{k+2}$.

3.2. In all the remaining cases we will show that $\bar{M}$ admits a flat metric with exactly $k$ conical singularities (Lemma 3.2). Then we remove these singularities by cutting $\bar{M}$ along simple closed curves which have only two critical points of geodesic curvature each (Lemma 3.3).

3.2.1. A cone is the surface generated by rays which emanate from the origin of $\mathbf{R}^{3}$ and pass through some closed rectifiable curve on $\mathbf{S}^{2}$. The length of this curve is the angle of the cone, and the origin is its vertex. A flat surface $\bar{M}$ with conical singularities is a metric space, homeomorphic to a 2-manifold, which is locally isometric to a neighborhood of the vertex of a cone. If the angle of this cone, for some point $p \in \bar{M}$, is not $2 \pi$, then we say $p$ is a conical singularity. Note that $\bar{M}$ is a smooth Riemannian manifold of zero curvature in the complement of these singular points. So if $\bar{M}$ has $k$ singularities of angles $\theta_{i}$, and $\chi$ is the Euler characteristic, then by the Gauss-Bonnet theorem, $\sum_{i=1}^{k}\left(-\theta_{i}\right)=2 \pi(\chi(\bar{M})-k)$ or

$$
\sum_{i=1}^{k}\left(2 \pi-\theta_{i}\right)=2 \pi \chi(\bar{M}) .
$$

Troyanov [27] has shown that the above condition is also sufficient for the existence of flat metrics with conical singularities of prescribed angles. This result quickly yields the following lemma. We include, however, a more direct proof which illustrates how explicit examples may be constructed. It follows from (11) that the lower bounds for $k$ below are sharp. 
Lemma 3.2. Let $\bar{M}$ be a closed surface, and suppose $k(\bar{M}) \geq 3,2$, 2, or 1 , according to whether $\chi(\bar{M})=2,1,0$, or $<0$, respectively. Then there exists a flat metric on $\bar{M}$ with exactly $k$ conical singularities.

Proof. If $\chi(\bar{M})=2$, i.e., $\bar{M}=\mathbf{S}^{2}$, take a planar convex polygon $P$ with $k$ vertices of angles $\theta_{i}<\pi$, and "double" it; i.e., identify the edges of $P$ with those of a congruent copy.

If $\chi(\bar{M})=1$, i.e., $\bar{M}=\mathbf{R P}^{2}$, let $P$ be a convex polygon with $k+1$ vertices of angles $\theta_{i}<\pi$ such that $\theta_{1}+\theta_{2} \neq \pi$ for a pair of vertices at the ends of a side $A$ of $P$. Then double $P$ with a "twist" along $A$.

If $\chi(\bar{M})=0$, i.e., $\bar{M}$ is a torus or a Klein bottle, take a rectangle $R$ and slightly "bend" one of its sides to add $k-1$ nontrivial vertices to its interior without changing the total length of that side or any other sides of $R$. Then glue the side of $R$ which has the new vertices to its opposite side, and identify the other two sides as well, possibly with a twist.

If $\chi(\bar{M})<0$, then $\bar{M}$ is homeomorphic to a planar convex polygon $P$ (the standard fundamental polygon of $\bar{M}$ ) with $2 n$ sides, where $n>2$, which are identified in pairs. Furthermore, all vertices of $P$ are identified with each other. Thus $P$ induces on $\bar{M}$ a flat metric with one cone point of angle $(2 n-2) \pi>4 \pi$. Furthe singularities may be added by bending one of the sides of $P$ to add $k-1$ vertices, as we described in the case of $\chi(\bar{M})=0$.

3.2.2. Now we remove the singularities $v_{i}$ of the surface $\bar{M}$ in the previous lemma by cutting $\bar{M}$ along simple closed curves $\gamma_{i}$, with only two critical points of curvature, which encircle $v_{i}$. More precisely, each $\gamma_{i}$ bounds an open disk $D_{i}$ which contains $v_{i}$. Furthermore, we can choose the radii of these disks so small that their closures are mutually disjoint. Then $M:=\bar{M}-\bigcup_{i=1}^{k} D_{i}$ is our desired flat surface. The curves we need are given by the following observation:

Lemma 3.3. Let $C$ be a cone with angle $\phi \neq 2 \pi$ and $\Gamma$ be a circle centered at the vertex of $C$. Then there exists a $\mathcal{C}^{\infty}$ perturbation of $\Gamma$ which has only two critical points of curvature, and each of its critical points is nondegenerate.

Proof. After a rescaling, we may suppose that $\Gamma$ has radius 1 and consider the following two cases:

Case I. If $\phi=2 n \pi$ (where $n \geq 2$ ), then $C$ may be identified with an $n$-sheeted branched covering of the plane with exactly one branch point at the origin $o$ of $\mathbf{R}^{2}$. Then, in polar coordinates, our desired curve is given by

$$
r_{\lambda}(\theta):=1-\lambda \cos \left(\frac{\theta}{n}\right),
$$

where $0 \leq \theta \leq 2 n \pi$ and $\lambda$ is a small constant. In particular, a computation shows that any $0<\lambda \leq 1 / 3$ would do. Figure 3 shows pictures of $r_{\lambda}$ for $n=2,3,4$ and $\lambda=1 / 3$. The curvature of $r_{\lambda}$ has only two critical points, which occur at $\theta=0$ and $\theta=n \pi$, and are both nondegenerate.

Case II. If $\phi \neq 2 n \pi$, we cut $C$ along one of the rays $r$ emanating from its vertex $v$ and develop it into the plane such that the image of $\Gamma$ may be parametrized as $\gamma_{0}(t)=(\cos (t), \sin (t)),-\phi / 2 \leq t \leq \phi / 2$. Then $v$ gets mapped to $o$ and $r$ gets identified with a pair of rays $r^{ \pm}$in $\mathbf{R}^{2}$, which emanate from $o$ and make angles of 

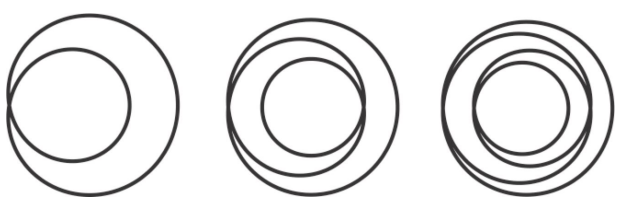

Figure 3

$\pm \phi / 2$ with the positive direction of the $x$-axis respectively. So, since $\phi \neq 2 n \pi, r^{ \pm}$ will not be parallel to the $x$-axis. Now set

$$
\kappa_{\lambda}(t):=1-\lambda \cos \left(\frac{2 \pi t}{\phi}\right) .
$$

Let $\gamma_{\lambda}(t)$ be the unit speed curve with prescribed curvature $\kappa_{\lambda}(t)$ and initial conditions $\gamma_{\lambda}(0)=(1,0)$ and $\gamma_{\lambda}^{\prime}(0)=(0,1)$. If $\theta_{\lambda}(t)$ indicates the angle between $\gamma_{\lambda}^{\prime}(t)$ and $\gamma_{\lambda}^{\prime}(0)$, then

$$
\theta_{\lambda}\left( \pm \frac{\phi}{2}\right)=\int_{0}^{ \pm \phi / 2} \theta_{\lambda}^{\prime}(t) d t=\int_{0}^{ \pm \phi / 2} \kappa_{\lambda}(t) d t= \pm \frac{\phi}{2}=\theta_{0}\left( \pm \frac{\phi}{2}\right) .
$$

So $\gamma_{\lambda}^{\prime}( \pm \phi / 2)=\gamma_{0}^{\prime}( \pm \phi / 2)$. Now, since $r^{ \pm}$are not parallel to the $x$-axis, we may translate $\gamma_{\lambda}$ parallel to the $x$-axis until we obtain a curve $\bar{\gamma}_{\lambda}:=\gamma_{\lambda}+(a(\lambda), 0)$ whose end points lie on $r^{ \pm}$. Then the ends of $\bar{\gamma}_{\lambda}$ will meet $r^{ \pm}$orthogonally. Since $\gamma_{0}$ is a radial graph over $o$, we may choose $\lambda$ so small that $\bar{\gamma}_{\lambda}$ is a graph over $o$ as well, since $\left\|\bar{\gamma}_{\lambda}-\gamma_{0}\right\|_{\mathcal{C}^{\infty}} \rightarrow 0$ as $\lambda \rightarrow 0$. So $\bar{\gamma}_{\lambda}$ corresponds to a simple closed $\mathcal{C}^{1}$ curve $\Gamma_{\lambda}$ in $C$ which encircles $v$. Finally, as we argued in Section 3.1.2, since the period of $\kappa_{\lambda}$ coincides with the length of $\Gamma_{\lambda}$, Lemma 3.1 shows that $\Gamma_{\lambda}$ is $\mathcal{C}^{\infty}$, and its curvature is given by $\kappa_{\lambda}$. So $\Gamma_{\lambda}$ will have only two critical points of curvature.

Note 3.4. The method of Section 3.1.2 may be generalized to construct flat surfaces of every nonzero genus with the aid of the family of unit speed curves $\gamma_{n}$ with prescribed curvature

$$
\kappa_{n}(t)=1-\frac{3}{4} \cos \left(\frac{t}{2 n-1}\right),
$$

where $-(2 n-1) \pi \leq t \leq(2 n-1) \pi$. Figure 4 shows $\gamma_{2}$ and its corresponding region,

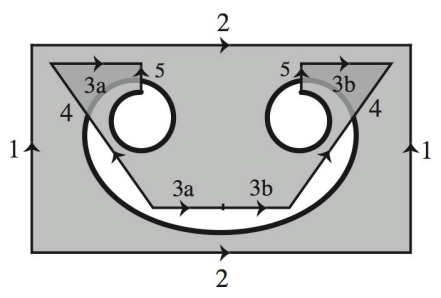

FigURE 4

whose sides may be identified in the manner indicated to yield a surface of genus 2. Similarly, each $\gamma_{n}$ may be used to construct an example of genus $n$. 


\section{Perturbations of Flat metrics}

If every critical point of geodesic curvature $\kappa$ on the boundary of a surface $M$ is nondegenerate, then the number of the critical points of $\kappa$ will be preserved under (small) $\mathcal{C}^{3}$-perturbations of the metric (since they induce $\mathcal{C}^{2}$ perturbations of $\kappa$ ). Thus applying the following result to flat surfaces we constructed in the previous section yields hyperbolic and elliptic metrics of small constant curvature on these surfaces with only two critical points of $\kappa$ on each of their boundary components. A rescaling then yields such metrics of any constant curvature. The $\mathcal{C}^{k}$ topology on the space of metrics on $M$ is defined by declaring that a pair of metrics $g$ and $h$ are $C^{k}$-close when their coefficients $g_{i j}$ and $h_{i j}$ are close up to their $k^{t h}$ derivative in local coordinates.

Proposition 4.1. Let $M$ be a compact surface with boundary and flat metric $g_{0}$. Then there exists a family $g_{\lambda}$ of Riemannian metrics on $M, \lambda \in(-\epsilon, \epsilon)$ for some $\epsilon>0$, such that $g_{\lambda}$ has constant curvature $\lambda$ and $\lambda \mapsto g_{\lambda}$ is continuous with respect to the $\mathcal{C}^{\infty}$ topology.

Proof. It is a basic topological fact that $M$ may be cut along a finite number of simple curves to obtain a surface $M^{\prime}$ which is homeomorphic to $\mathbf{S}^{2}$ minus a finite number of open disks. If we further cut $M^{\prime}$ along pairwise disjoint simple curve segments which connect each component of $\partial M$ to $\partial M^{\prime}-\partial M$, then we obtain a simply connected surface $M^{\prime \prime}$ with piecewise smooth boundary. Since $M^{\prime \prime}$ inherits a flat metric from $M$, it may be developed into $\mathbf{R}^{2}$ by Lemma 2.2 (Figures 1 and 2 depict examples of such regions).

Thus we obtain a piecewise smooth closed planar curve $\Gamma$ bounding an isometric immersion of $M^{\prime \prime}$. Let $p$ be a point of $\Gamma$ which lies in the interior of an edge of $M^{\prime \prime}$ which is not a segment of $\partial M$. Parameterize $\Gamma$ by arclength to obtain a piecewise smooth periodic curve $\gamma: \mathbf{R} / L \rightarrow \mathbf{R}^{2}$ with $\gamma(n L)=p$. Next let us perturb the standard metric of $\mathbf{R}^{2}$, on a compact set containing $\Gamma$, by setting

$$
\left(g_{\lambda}\right)_{i j}(x):=\frac{\delta_{i j}}{\left(1+\frac{\lambda}{4}\|x\|^{2}\right)^{2}} .
$$

As was already known to Riemann [23], $g_{\lambda}$ has constant curvature $\lambda$. Now define $\gamma_{\lambda}: \mathbf{R} \rightarrow \mathbf{R}^{2}$ to be the piecewise smooth curve parametrized by arclength, with respect to $g_{\lambda}$, such that $\gamma_{\lambda}(0)=\gamma(0), \gamma_{\lambda}^{\prime}(0)$ is parallel to $\gamma^{\prime}(0)$, each edge of $\gamma_{\lambda}$ has the same curvature function as the corresponding edge of $\gamma$, and the angle at each vertex of $\gamma_{\lambda}$ is equal to the angle of the corresponding vertex of $\gamma$. Note that $\lambda \mapsto \gamma_{\lambda}$ is continuous with respect to the $\mathcal{C}^{\infty}$-norm, since $\gamma_{\lambda}$ is the unique solution to an ODE, as we will show in Lemma 4.2 below, whose coefficients are determined by $g_{\lambda}$.

We are going to smoothly "close up" $\gamma_{\lambda}$ by the following perturbation. Choose $\delta>0$ so small that $\gamma([-\delta, 0])$ lies entirely in the interior of the edge of $\gamma$ containing $p$, and let $\phi:[0, L] \rightarrow \mathbf{R}$ be any smooth nonincreasing function with $\phi \equiv 1$ on $[0, L-\delta]$ and $\phi \equiv 0$ near $L$. Define $\bar{\gamma}_{\lambda}:[0, L] \rightarrow \mathbf{R}^{2}$ by

$$
\bar{\gamma}_{\lambda}(t):=\phi(t) \gamma_{\lambda}(t)+(1-\phi(t)) \gamma_{\lambda}(t-L) .
$$

It is easy to check that $\bar{\gamma}_{\lambda}$ is a smooth closed curve. Further, $\bar{\gamma}_{\lambda}$ is $\mathcal{C}^{\infty}$-close to $\gamma$, because, as $\lambda \rightarrow 0, \gamma_{\lambda} \rightarrow \gamma$; therefore, since $\gamma$ is $L$-periodic,

$$
\bar{\gamma}_{\lambda}(t) \longrightarrow \phi(t) \gamma(t)+(1-\phi(t)) \gamma(t-L)=\gamma(t) .
$$


In particular, for small $\lambda, \bar{\gamma}_{\lambda}$ will bound an immersed surface, say $M_{\lambda}^{\prime \prime}$, which will be homeomorphic to $M^{\prime \prime}$, as we will verify in Lemma 4.3 below.

We claim that gluing the sides of $M_{\lambda}^{\prime \prime}$ (in the same fashion as the corresponding sides of $M^{\prime \prime}$ ) yields the desired perturbation of $M$. To see this note that if $A$ and $B$ are a pair of sides of $M^{\prime \prime}$ which are to be glued, then the sum of the geodesic curvatures at the corresponding points of $A$ and $B$ are zero. But, by construction, each side of $M_{\lambda}^{\prime \prime}$, which is not a segment of $\partial M$, has the same geodesic curvature as the corresponding side of $M^{\prime \prime}$. Thus gluing the sides of $M_{\lambda}^{\prime \prime}$ yields a smooth surface (here we also use the fact that the sum of the angles at all corners $M_{\lambda}^{\prime \prime}$ are $2 \pi$, since all angles are the same as those in $M^{\prime \prime}$ ). This smooth surface, which is homeomorphic to $M$, will carry a metric which is locally the same as $g_{\lambda}$ and therefore will have constant curvature $\lambda$, as desired.

It only remains to establish the following two lemmas. The first one of these is the Riemannian version of the fundamental theorem of planar curves. The proof, which follows from basic ODE theory, is a generalization of the well-known argument for the existence and uniqueness of geodesics. So it will be omitted.

Lemma 4.2. Let $M$ be a complete oriented $\mathcal{C}^{\infty}$ Riemannian surface, $p \in M$, and $u \in T_{p} M$ be a unit vector. Suppose that we are given a $\mathcal{C}^{\infty}$ function $\kappa: I \rightarrow \mathbf{R}$, for some open interval $I \subset \mathbf{R}$ with $0 \in I$. Then there exists a unique unit speed $\mathcal{C}^{\infty}$ curve $\gamma: I \rightarrow M$ with $\gamma(0)=p, \gamma^{\prime}(0)=u$, and geodesic curvature $\kappa(t)$.

A piecewise $\mathcal{C}^{k}$ curve $\gamma: \mathbf{S}^{1} \rightarrow \mathbf{R}^{2}$ is one which is $\mathcal{C}^{k}$ in the complement of a finite number of points, and its right and left derivatives exist everywhere up to order $k$. The space $X$ of these curves is topologized by stipulating that $\gamma_{0}, \gamma_{1} \in X$ are $\mathcal{C}^{k}$-close provided that they are $\mathcal{C}^{0}$-close, and their left and right derivatives up to order $k$ are close as well.

Lemma 4.3. In the space of piecewise $\mathcal{C}^{1}$ closed curves $\gamma: \mathbf{S}^{1} \rightarrow \mathbf{R}^{2}$, the set of curves which bound compact immersed surfaces of a given topological type is open.

Proof. Let $M$ be a compact surface with connected boundary, and let $f_{0}: M \rightarrow \mathbf{R}^{2}$ be an immersion bounded by a closed piecewise $\mathcal{C}^{1}$ curve $\gamma_{0}$; i.e., $\gamma_{0}$ is a reparameterization of $f_{0}$ restricted to $\partial M$. Recall that $M$ may be extended to a manifold without boundary $\bar{M}$ (e.g., by doubling $M$ ). Since $\partial M$ is compact, there exists a tubular neighborhood $U$ of $\partial M$ in $\bar{M}$ such that $f_{0}$ may be extended to $U$. We may suppose that $\bar{M}=M \cup U$ and endow $\bar{M}$ with the pull-back metric induced by $f_{0}$. Now let $\epsilon>0$ be so small that $f_{0}$ is an embedding on an $\epsilon$-ball centered at any point of $\partial M$. Next suppose that a closed piecewise $\mathcal{C}^{1}$ curve $\gamma_{1}$ is so close to $\gamma_{0}$ that $\gamma_{1}(t)$ lies within an $\epsilon$-ball of $\gamma_{0}(t)$ for all $t$. This will allow us to pull back $\gamma_{1}$ to $\bar{M}$ via $f_{0}$. Let $\bar{\gamma}_{0}$ and $\bar{\gamma}_{1}$ be the pull-backs of these curves. Then $\bar{\gamma}_{0}$ and $\bar{\gamma}_{1}$ will be $\mathcal{C}^{1}$-close if $\gamma_{0}$ and $\gamma_{1}$ are $\mathcal{C}^{1}$ close. So, since $\bar{\gamma}_{0}$ is simple, we may assume that $\bar{\gamma}_{1}$ is simple as well; indeed, they are isotopic via the pull-back of the homotopy $\gamma_{\lambda}(t):=\lambda \gamma_{1}(t)+(1-\lambda) \gamma_{0}(t)$ when $\gamma_{0}$ and $\gamma_{1}$ are $\mathcal{C}^{1}$-close. Now it follows from the isotopy extension lemma [10 that $\bar{\gamma}_{1}$ bounds a region in $\bar{M}$ which is homeomorphic to the region bounded by $\bar{\gamma}_{0}$, which is just $M$. So the restriction of $f_{0}$ to the region bounded by $\bar{\gamma}_{1}$ yields an immersed surface which is homeomorphic to $M$ and is bounded by $\gamma_{1}$. 


\section{ACKNOWLEDGEMENTS}

The author thanks Jason Cantarella, Ralph Howard, Joachim Lohkamp, Rick Schoen, Serge Tabachnikov, Michael Wolf, and especially Rob Kusner for useful communications.

\section{REFERENCES}

[1] R. Benedetti and C. Petronio. Lectures on hyperbolic geometry. Universitext. Springer-Verlag, Berlin, 1992. MR.1219310 (94e:57015)

[2] S. Brendle. Curvature flows on surfaces with boundary. Math. Ann., 324(3):491-519, 2002. MR:1938456 (2003j:53103)

[3] G. Cairns, M. Ozdemir, and E.-H. Tjaden. A counterexample to a conjecture of U. Pinkall. Topology, 31(3):557-558, 1992. MR.1174258 (93d:53004)

[4] S. I. R. Costa and M. Firer. Four-or-more-vertex theorems for constant curvature manifolds. In Real and complex singularities (São Carlos, 1998), volume 412 of Chapman \& Hall/CRC Res. Notes Math., pages 164-172. Chapman \& Hall/CRC, Boca Raton, FL, 2000. MR 1715702 (2001d:51013)

[5] D. DeTurck, H. Gluck, D. Pomerleano, and D. S. Vick. The four vertex theorem and its converse. Notices Amer. Math. Soc., 54(2):192-207, 2007. MR2285124 (2007k:53003)

[6] M. Ghomi. Vertices of closed curves in Riemannian surfaces. Preprint available at www.math.gatech.edu/ ghomi. arXiv:1006.4182v1

[7] M. Ghomi and M. Kossowski. $h$-principles for hypersurfaces with prescribed principal curvatures and directions. Trans. Amer. Math. Soc., 358(10):4379-4393 (electronic), 2006. MR2231382 (2007c:53072)

[8] H. Gluck. The generalized Minkowski problem in differential geometry in the large. Ann. of Math. (2), 96:245-276, 1972. MR0309021 (46:8132)

[9] B. Guan and J. Spruck. Locally convex hypersurfaces of constant curvature with boundary. Comm. Pure Appl. Math., 57(10):1311-1331, 2004. MR2069725 (2005d:53097)

[10] M. W. Hirsch. Differential topology. Springer-Verlag, New York, 1994. Corrected reprint of the 1976 original. MR 1336822 (96c:57001)

[11] S. B. Jackson. Vertices for plane curves. Bull. Amer. Math. Soc., 50:564-478, 1944. MR0010992 (6:100e)

[12] S. B. Jackson. The four-vertex theorem for surfaces of constant curvature. Amer. J. Math., 67:563-582, 1945. MR0014257 (7:259h)

[13] A. Kneser. Bemerkungen über die anzahl der extrema des krümmung auf geschlossenen kurven und über verwandte fragen in einer night eucklidischen geometrie. In Festschrift Heinrich Weber, pages 170-180. Teubner, 1912.

[14] M. Maeda. The four-or-more vertex theorems in 2-dimensional space forms. Nat. Sci. J. Fac. Educ. Hum. Sci. Yokohama Natl. Univ., (1):43-46, 1998. MR1710269 (2001a:53023)

[15] H. Masur and S. Tabachnikov. Rational billiards and flat structures. In Handbook of dynamical systems, Vol. 1A, pages 1015-1089. North-Holland, Amsterdam, 2002. MR.1928530 (2003j:37002)

[16] S. Mukhopadhyaya. New methods in the geometry of a plane arc. Bull. Calcutta Math. Soc. I, pages 31-37, 1909.

[17] B. Osgood, R. Phillips, and P. Sarnak. Extremals of determinants of Laplacians. J. Funct. Anal., 80(1):148-211, 1988. MR960228 (90d:58159)

[18] V. Ovsienko and S. Tabachnikov. Projective differential geometry old and new, volume 165 of Cambridge Tracts in Mathematics. Cambridge University Press, Cambridge, 2005. From the Schwarzian derivative to the cohomology of diffeomorphism groups. MR2177471 (2007b:53017)

[19] U. Pinkall. On the four-vertex theorem. Aequationes Math., 34(2-3):221-230, 1987. MR921101 (89a:53007)

[20] A. V. Pogorelov. Extrinsic geometry of convex surfaces. Translated from the Russian by Israel Program for Scientific Translations, Translations of Mathematical Monographs, Vol. 35. American Mathematical Society, Providence, RI, 1973. MR0346714 (49:11439)

[21] J. G. Ratcliffe. Foundations of hyperbolic manifolds, volume 149 of Graduate Texts in Mathematics. Springer, New York, second edition, 2006. MR2249478 (2007d:57029) 
[22] E. Raphaël, J.-M. di Meglio, M. Berger and E. Calabi. Convex particles at interfaces, J. Phys. I France 2:571-579, 1992.

[23] M. Spivak. A comprehensive introduction to differential geometry. Vol. II. Publish or Perish Inc., Wilmington, Del., second edition, 1979. MR0532831 (82g:53003b)

[24] G. Thorbergsson and M. Umehara. A unified approach to the four vertex theorems. II. In Differential and symplectic topology of knots and curves, volume 190 of Amer. Math. Soc. Transl. Ser. 2, pages 229-252. Amer. Math. Soc., Providence, RI, 1999. MR.1738398(2001f:53009)

[25] W. P. Thurston. Shapes of polyhedra and triangulations of the sphere. In The Epstein birthday schrift, volume 1 of Geom. Topol. Monogr., pages 511-549 (electronic). Geom. Topol. Publ., Coventry, 1998. MR $1668340(2000 \mathrm{~b}: 57026)$

[26] M. Troyanov. Les surfaces euclidiennes à singularités coniques. Enseign. Math. (2), 32(1-2): 79-94, 1986. MR850552 (87i:30079)

[27] M. Troyanov. On the moduli space of singular Euclidean surfaces. In Handbook of Teichmüller theory. Vol. I, volume 11 of IRMA Lect. Math. Theor. Phys., pages 507-540. Eur. Math. Soc., Zürich, 2007. MR22349679 (2009b:57041)

[28] N. S. Trudinger and X.-J. Wang. On locally convex hypersurfaces with boundary. J. Reine Angew. Math., 551:11-32, 2002. MR 1932171(2004b:58017)

[29] M. Umehara. 6-vertex theorem for closed planar curve which bounds an immersed surface with nonzero genus. Nagoya Math. J., 134:75-89, 1994. MR.1280654 (95e:53007)

[30] M. Umehara. A unified approach to the four vertex theorems. I. In Differential and symplectic topology of knots and curves, volume 190 of Amer. Math. Soc. Transl. Ser. 2, pages 185-228. Amer. Math. Soc., Providence, RI, 1999. MR:1738397 (2001f:53008)

School of Mathematics, Georgia Institute of Technology, Atlanta, Georgia 30332

E-mail address: ghomi@math.gatech.edu

$U R L$ : www . math. gatech.edu/ ghomi 